\title{
The Influence of X-ray Counting Statistics on Trace Analysis and Spatial Resolution
}

\author{
Eric Lifshin* and Raynald Gauvin** \\ * University at Albany, School of Nanosciences and Nanoengineering, Albany, NY, 12203 \\ ** McGill University, Department of Mining, Metals and Materials, Montreal, Quebec, Canada \\ H3A2B2
}

It is well recognized that under proper circumstances the relative accuracy of quantitative electron microprobe analysis can be better than 2\% [1]. Critical requirements include the use of well prepared polished samples, good standards, stable electron beam and x-ray detector operation, a small excitation volume relative to the phase of interest, generally more than $10 \%$ of the element of interest, minimal spectral interferences, and a good quantitative model containing accurate parameters. Precision, on the other hand, is determined by the variability of repeated measurements under what should be constant operating conditions. The factors affecting precision include stage and spectrometer reproducibility, noise in the electron beam, sample inhomogeneity and the inherent randomness of the x-ray emission process itself. The latter is described by x-ray counting statistics. Under the conditions stated above for the high accuracy case it is usually not difficult to acquire sufficient counts from samples and standards in reasonable times (100 seconds or less) to have the contribution to the precision from x-ray counting statistics be less than $2 \%$. Here precision is given by the $95 \%$ confidence interval defined by + or $-1.96 s_{c}$ where $s_{c}$ is the standard deviation in the composition [2,3]. This level is generally possible with either energy dispersive spectrometry (EDS) or wavelength dispersive spectrometery (WDS) since measurable count rates of at least several thousand counts per second are often possible for many pure elements. When sufficient counting times are available then precision for the high accuracy case may often be limited by factors other than counting statistics and $s_{c}$ should be determined experimentally.

There are, however, frequently encountered circumstances where the precision due to counting statistics can be much larger than $2 \%$ and counting statistics may be the main factor limiting precision. These include the analysis of thin films and fine phases as well as trace element determination. In these cases the count rates may be so low and close to background levels that it is not always practical or even possible to count long enough to accumulate the needed numbers of counts because of drift, contamination or the lack of long term instrument availability. Monte Carlo modeling [4] optimized to give accurate characteristic line and continuum intensities can be very effectively used in conjunction with detector collection efficiency data serves as an excellent way to predict precision as a function of a variety of experimental parameters. These include SEM (or electron microprobe) operating conditions, $\mathrm{x}$-ray detector operating conditions, sample composition and sample morphology including thickness for thin films or phase size for structures with lateral dimensions smaller than the electron beam excited volume. Fig. 1 is an example of a 95\% confidence plot as a function of electron beam voltage for a WDS system measuring aluminum $\mathrm{K}_{\alpha}$ in an AlGaAs sample. The different curves show how beam current and counting time influence precision. 
Beam voltage was chosen as an independent variable because it must be reduced to decrease the excitation volume as shown in Fig. 2 illustrating the Anderson-Hasler range [5] for the production of $\mathrm{Al} \mathrm{K} \alpha \mathrm{x}$-rays in both $\mathrm{Al}$ and AlGaAs. A more detailed three dimensional picture of the excitation volume can be obtained with Monte Carlo calculations which also show the effect of electron beam diameter. X-ray intensity is directly proportional to probe current, and probe current decreases rapidly with probe diameter for all the different types of electron sources used. The probe current also decreases with beam voltage for a given probe size. Obtaining a small probe $(30 \mathrm{~nm}<)$ with stable current of $1 \mathrm{nA}$ at low voltages $(5 \mathrm{KV})$ can be a real challenge, but can be done with Schottky sources. In addition, the excitation efficiency of x-ray lines drops as beam voltage approaches the line excitation energy. All of these factors can combine to limit x-ray emission under conditions required for high spatial resolution, however expected performance and optimum conditions for each experimental situation can be defined by modeling. A similar approach has been developed for thin film and trace analysis. The authors wish to acknowledge the support of the MARCO and DARPA sponsored Interconnect Focus Center.

\section{References}

[1] J. Pouchou and J Pichoir, Electron Probe Quantitation, Heinrich and Newbury eds., Plenum Press, 31-75 (1991)

[2] T. O. Ziebold, Anal. Chem., 39, 858-861 (1967)

[3] E. Lifshin, N. Doganaksoy, J. Sirois and R. Gauvin, Microsc. Microanal, 4 598-604 (1999)

[4] E. Lifshin and R. Gauvin, Microsc and Microanal 4,232-233 (1998)

[5] C. A. Anderson and M. F. Hasler, Proc. $4^{\text {th }}$ Intl. Conf. X-ray Optics and Microanalysis, Hermann Paris, p 310 (1966)

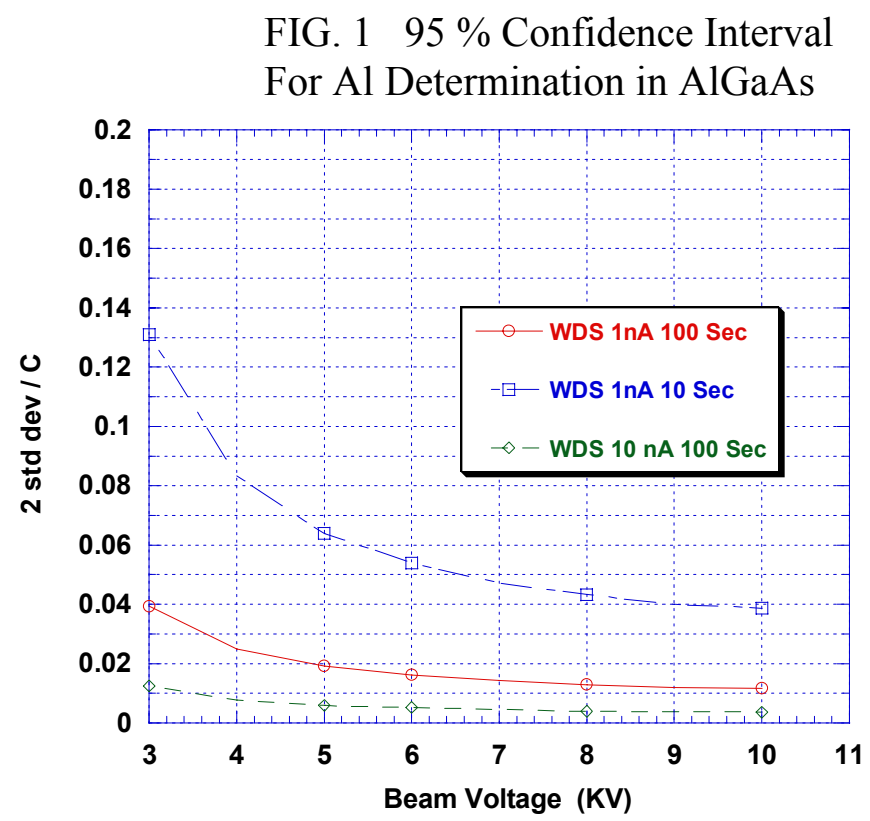

FIG. 2 Anderson - Hasler

Range for $\mathrm{Al} \mathrm{K} \mathrm{K}_{\alpha} \mathrm{X}$-ray

Production in $\mathrm{Al}$ and $\mathrm{AlGaAs}$

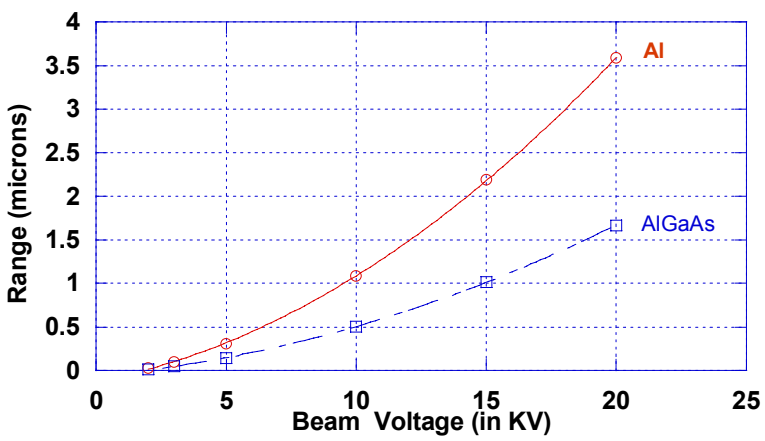

